University of San Diego

Digital USD

Spring 5-30-2021

\title{
Deprescribing in the Hospice Population, Who is Eligible and Who is Agreeable: A Quality Improvement Project
}

Ashlee Nicole Smith

University of San Diego, ashleesmith@sandiego.edu

Follow this and additional works at: https://digital.sandiego.edu/dnp

Part of the Nursing Commons, Other Pharmacy and Pharmaceutical Sciences Commons, and the Palliative Care Commons

\section{Digital USD Citation}

Smith, Ashlee Nicole, "Deprescribing in the Hospice Population, Who is Eligible and Who is Agreeable: A Quality Improvement Project" (2021). Doctor of Nursing Practice Final Manuscripts. 154.

https://digital.sandiego.edu/dnp/154

This Doctor of Nursing Practice Final Manuscript is brought to you for free and open access by the Theses and Dissertations at Digital USD. It has been accepted for inclusion in Doctor of Nursing Practice Final Manuscripts by an authorized administrator of Digital USD. For more information, please contact digital@sandiego.edu. 
Deprescribing in the Hospice Population, Who is Eligible and Who is Agreeable: A Quality Improvement Project

UNIVERSITY OF SAN DIEGO

Hahn School of Nursing and Health Science Beyster Institute of Nursing

DOCTOR OF NURSING PRACTICE PORTFOLIO

by

Ashlee Nicole Smith

A portfolio presented to the

FACULTY OF THE HAHN SCHOOL OF NURSING AND HEALTH SCIENCE UNIVERSITY OF SAN DIEGO

In partial fulfillment of the requirements for the degree

DOCTOR OF NURSING PRACTICE

May 2021 


\section{Table of Contents}

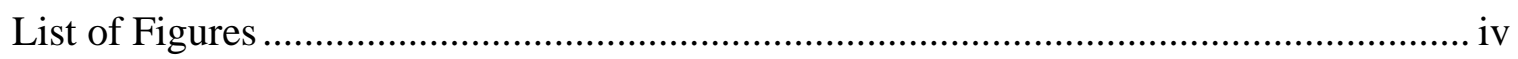

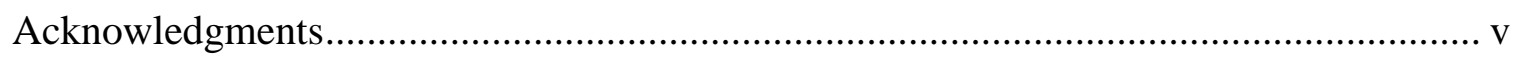

Deprescribing in the Hospice Population, Who is Eligible and Who is Agreeable: A

Quality Improvement Project........................................................................ 1

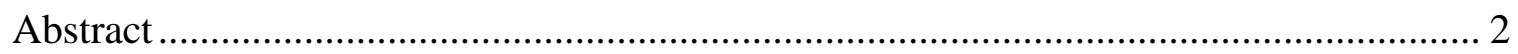

Background and Significance: Polypharmacy and Deprescribing in Hospice ....... 3

Evidence for the Project.......................................................................... 4

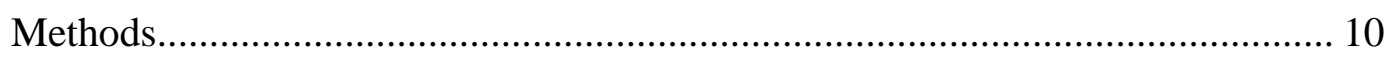

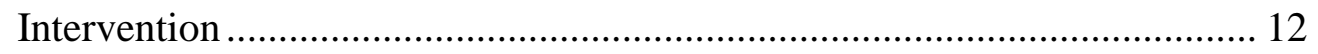

Measurable Outcomes........................................................................ 13

Data Collection .......................................................................... 13

Protection of Human Subjects .................................................................. 14

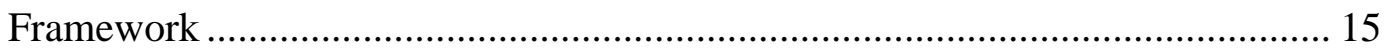

DMAIC Framework ....................................................................... 15

Change Acceleration Process Model ..................................................... 15

Meleis' Transitions Theory ............................................................. 16

Holmes' Model of Deprescribing ..................................................... 17

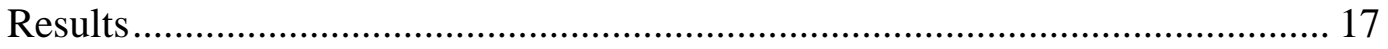

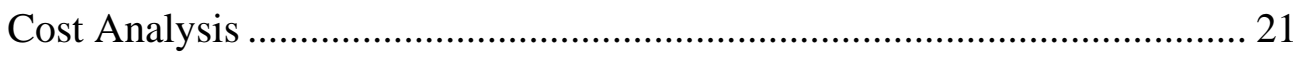

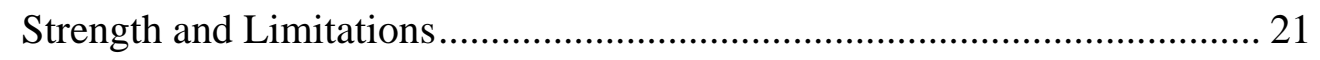

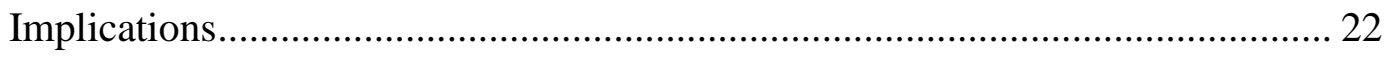


Conclusion .

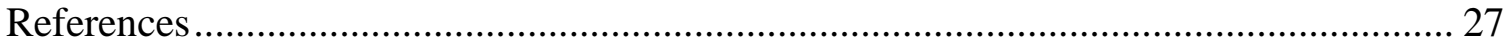




\section{List of Figures}

Figure 1 Percentage of Target Medications Eligible to Deprescribe ...............................21

Figure 2 Deprescribing at Different Intervals ......................................................21

Figure 3 Primary Hospice Admitting Diagnoses ...................................................22 


\section{Acknowledgments}

I would like to thank my faculty advisor Dr. Caroline Etland for her guidance in completing this project. Thank you to my clinical advisor Dr. Tracie Gadler for her expertise and unwavering assistance to make this project feasible. To my grandma, Judy, for counting down the days for me. And lastly, to my husband Jack, for his love and patience in enduring this journey with me. 

DEPRESCRIBING IN THE HOSPICE POPULATION, WHO IS ELIGIBLE AND WHO IS

AGREEABLE: A QUALITY IMPROVEMENT PROJECT

Deprescribing in the Hospice Population, Who is Eligible and Who is Agreeable: A Quality

Improvement Project

Ashlee N. Smith

University of San Diego 


\begin{abstract}
Current evidence recommends and supports deprescribing medications to reduce polypharmacy in the hospice population. The project aim was to determine accurate percentages of deprescribing at three-time periods: before hospice admission, at hospice enrollment, and four weeks post hospice enrollment. This project was a retrospective review of the medication administration record (MAR) of patients admitted into Sharp Hospice from a Sharp Healthcare facility. Sharp Hospice, in California, USA, admitted 164 patients during August 2020. Of those, 34 patients were eligible for inclusion in this retrospective quality improvement review. Prior to discharge from at a Sharp facility, the percentage of target medications on the inpatient medication record equaled $80.5 \%$ for antihypertensives, $61.1 \%$ for statins, and $22.2 \%$ for oral diabetic medications for the sample. At hospice admission, $61.0 \%$ of these patients were appropriately deprescribed, $13.8 \%$ of patients were partially deprescribed, meaning some eligible medications were removed, and $25 \%$ of patients were not deprescribed. $64 \%$ of patients were appropriately deprescribed target medications during the first four weeks of hospice enrollment. The results confirmed medication deprescribing is occurring but also identified missed opportunities for deprescribing in Sharp Hospice. These conclusions are beneficial and applicable to the next phase of project implementation to improve the organization's deprescribing percentages.
\end{abstract}

Keywords: Polypharmacy, deprescribing, hospice, end of life 
DEPRESCRIBING IN THE HOSPICE POPULATION, WHO IS ELIGIBLE AND WHO IS AGREEABLE: A QUALITY IMPROVEMENT PROJECT

Deprescribing in the Hospice Population, Who is Eligible and Who is Agreeable: A Quality Improvement Project

\section{Background and Significance: Polypharmacy and Deprescribing in Hospice}

Hospice clinicians are challenged with tertiary prevention of many complex and endstage disease processes, which have resulted in a laundry list of medications. Successful management of the hospice patient includes careful consideration of each medication previously prescribed to determine if it is necessary for the current goals of care, emphasizing symptom relief. Polypharmacy, defined as more medications than medically necessary, is not confined to the elderly, but they are most at risk of potentially inappropriate medications (PIMs) (Masnoon, 2017). Deprescribing is a term used to eliminate medications after a careful risk versus benefit analysis for each patient (Thompson, 2019). Often, treatment of comorbid conditions results in a prescribing cascade, leading to multiple additional medications. Although medical providers are taught about medication side effects throughout their education, associating polypharmacy with clinical symptoms is not always high on the differential diagnosis list. Research has suggested $29-51 \%$ of preventive medications such as aspirin, anti-hypertensives, and statins are continued in patients with a limited life expectancy, even though the timeframe of any potential benefits for these medications may be far longer than a patient's expected survival (Pruskowski, 2016).

Deprescribing may be underutilized for hospice patients which can result in unmet goals of care, specifically the reduction pain and suffering at the end of life. It is imperative to bridge the gap regarding the benefits of deprescribing and the reduction of polypharmacy to hospice patients and their families and promote a routine deprescribing plan within hospices.

The idea began in 2019 after sitting in on several weekly interdisciplinary team meetings in Sharp Hospice. This team included a member from each service in the hospice agency, 
including the clinical pharmacist, nurse practitioners, physicians, social workers, nurses, and chaplains. During this time, the team completed an individual chart review for each patient and discussed medications exhaustively, determining to continue to or to deprescribe. Often, this process was not time-efficient and resulted in miscommunication between clinicians, outlining the scope of the problem. The project goal was to determine how efficient current deprescribing practice was by assessing the percentage of target medication deprescribed at different time intervals. Target medications were identified during the interdisciplinary team meetings for inclusion and finalized after a literature review and synthesis of the evidence. The identified outcome was deprescribing being completed on patients, yet with a realization there was no standardized process which could result in some missed opportunities.

\section{Evidence for the Project}

Polypharmacy is a substantial problem in the elderly population and specifically with hospice patients. Deprescribing of certain medications during the end of life period is beneficial and appropriate for this patient population which has been demonstrated as level one evidence according to the Johns Hopkins Model (Cooper et.al., 2015). There is a multitude of additional level two and three evidence concluding deprescribing with a limited life expectancy is not only beneficial but reduces the risk of harm (Chau, et al., 2016; Schnecker et al., 2019). The following databases were searched for evidence: CINAHL, PubMed, EBSCOhost. Terms searched included: "Deprescribing AND Hospice," "Deprescribing AND Hypertensive Medications," “Deprescribing AND Statins,” Deprescribing AND Aspirin," "Deprescribing AND Hypoglycemics," "Deprescribing AND Cost Reduction," "Deprescribing AND Polypharmacy", "Deprescribing AND Geriatrics", "Polypharmacy AND Hospice”. After completing a literature review to acquire the current evidence, the scope of the problem was better understood. 
DEPRESCRIBING IN THE HOSPICE POPULATION, WHO IS ELIGIBLE AND WHO IS AGREEABLE: A QUALITY IMPROVEMENT PROJECT

\section{Metabolic Changes with Aging}

As the body ages, the pharmacokinetics of drug absorption, metabolism, and excretion becomes impaired, which translates to a need for a reduced dose to achieve the medication's same effect. The gastrointestinal system's ability to absorb changes with age and multiple medications cause alterations in absorption bioavailability, inducing the effects of polypharmacy. Due to inadequate water, fat, and protein concentrations in malnourished and dehydrated hospice patients, drug distribution is affected, and there is a greater risk of developing toxicity (Malone, 2019). Drug metabolism, which occurs primarily in the liver's cytochrome P (CYP) system, is also reduced. Elimination of drugs is most affected by the natural pharmacokinetic changes associated with age. Renal function declines steadily as aging occurs, even in the absence of disease, creating an extended half-life, allowing the drug to remain bioavailable in the body (Malone, 2019). For example, as renal function declines and drug excretion is prolonged, the half-life of glipizide medications, specifically Metformin, increases leading to a higher occurrence of hypoglycemia. (American Diabetic Association, 2021; Jeffreys, 2015). These pharmacokinetic and physiological changes have significant importance in a deprescribing discussion in the hospice population as the mean age of patients included in this analysis was 76 years old.

Concurrently with advancing age, the number of medications prescribed increases due to worsening of existing conditions and new disease processes. This combination of taking multiple medications and pharmacokinetics changes creates an environment for increased risk of adverse drug events, morbidity, and mortality. According to a population-based study, the average number of medications prescribed to an elderly patient over the age of 65 is more than five medications, and the risk of an adverse drug event is over $88 \%$ (Chau et al., 2016). Many elderly 
patients are at higher risk of an adverse drug reaction, and clinicians should take a standardized approach to reduce polypharmacy. Another specific example of polypharmacy leading to drugdrug interaction is concurrent use of diabetic agents and aspirin, which can inhibit desired hypoglycemia (Frechen et al., 2012). Polypharmacy is inevitable in the elderly population and increased in the hospice population without a comprehensive medication review and discontinuation plan.

\section{Deprescribing Target Medications}

Understanding which medications are appropriate to deprescribe requires evaluating the expected life span remaining, risk of an adverse event outweighing the benefits, and the current goals of care (Bukowy \& Teso, n.d.). Deprescribing medications is an essential aspect of care for all patients, but there is an increased emphasis on patients enrolled in hospice services as the medication's time to benefit may be greater than life expectancy. The topic of deprescribing may seem straightforward to many advanced clinicians, yet the simple act of discontinuing medications may present difficulties to patients and their families. A common misunderstanding due to lack of education could occur, as they may perceive deprescribing in hospice as hastening a loved one's death. This complicated emotional and psychological time during the end of life presents unique challenges when providing education. As end-of-life approaches, most of the patient's medications are not valuable or relevant to the current goals of care and may cause unwanted side effects and drug-drug interactions. Although the evidence supports routine deprescribing in the hospice population, it is essential to provide patients and families with education regarding the benefits. This retrospective analysis of deprescribing rates in Sharp Hospice is designed to prepare for phased implementation and assessment of barriers hindering education and implementation, as the next feasible step is to remove such barriers. 
A retrospective study evaluated the current prescribing and deprescribing practices for patients with palliative care services (McLean et al., 2013). The study reviewed prescribed medications to treat the most common comorbidities such as cardiovascular disease, ischemic heart disease, and dyslipidemia. According to the investigators, the number of medications during the last few weeks of life increased dramatically, with a mean total of 10 medications in the last week of life. Yet, there was little documentation found regarding deprescribing during this time. The authors also noted limited evidence regarding the burden of polypharmacy at the end of life. However, there is significant evidence supporting the benefits of deprescribing at the end of life, and there is a need for a standardized guideline to streamline the process and effectively deprescribe.

According to Kutner, et al. (2015), in a randomized controlled clinical trial (RCT) of endof-life patients and discontinuing statin therapy, removing the statin was safe and beneficial in terms of quality of life and reduced pill burden. This study resulted in a reduction of multiple complaints in the group who discontinued the statin. The symptoms, including muscle spasms, reduced strength, and exercise intolerance, were decreased in the arm of patients who discontinued statin therapy as compared to the group that continued therapy. One of the significant driving factors, which evidence has found to be valid and reliable, is the changes in pharmacokinetics, specifically metabolism, limiting the full potential benefit of statin therapy. Discussion with the patient and their families regarding the benefit versus burden of statin therapy with a decreased survival time included information that continued use would not result in a better outcome. However, informing patients and families of the physiologic benefits may not be convincing enough for the patient to agree to deprescribing. Explaining that a reduction in pill burden with no apparent harmful effects on life expectancy and reduced monthly costs could 
help persuade the patient to agree to eliminate statin therapy. Another RCT including 381 patients with limited life expectancy concluded no difference in 60-day mortality or cardiovascular events in one month to one year with statin discontinuation (Bukowy \& Teso, n.d.). Other beneficial aspects found were the improved quality of life for the hospice patient and reduced medication cost for the group who had medications discontinued as compared to the group who continued statin therapy.

Diabetes management for patients with a limited prognosis is not the same treatment plan for patients with an expected lifespan of many years. According to the American Diabetes Association, the Type 2 diabetic hospice patient should have a management goal of reducing the incidence of hypoglycemia and symptomatic hyperglycemia (2021). Once end-organ damage occurs, multiple agents will require a dose reduction or elimination to prevent polypharmacy and adverse drug reactions (American Diabetes Association, 2021). The Canadian Deprescribing Network has developed several research-based guidelines detailing how to achieve successful and safe deprescribing of anti-hyperglycemic agents, which are beneficial for applying in the clinical practice (Deprescribing Network, 2020). In a Cochrane meta-analysis review of interventions to reduce polypharmacy in the elderly, 12 studies were reviewed, concluding there is a lack of interventional research to improve polypharmacy (Cooper et al., 2015). Stopping diabetic medications in patients with an anticipated survival of weeks was deemed safe to prevent a hypoglycemic event with the rapid oral intake reduction. Although hyperglycemia may occur, it is unlikely the Type 2 diabetic hospice patient will go into a hyperosmolar hyperglycemic state due to the erratic eating patterns during end-of-life. Discussion with families and patients that tight glycemic control is not appropriate during the end-of-life period as the 
DEPRESCRIBING IN THE HOSPICE POPULATION, WHO IS ELIGIBLE AND WHO IS AGREEABLE: A QUALITY IMPROVEMENT PROJECT

goal is not to prevent the long-term complications of diabetes (secondary prevention) but to avoid hypoglycemia (Jeffreys et al., 2015).

Similar to abandoning strict blood glucose control, loosening blood pressure control with the hospice patient is safe. The goal for blood pressure should be less than 180/90 as symptomatic hypertension is associated with increases above this number. In most cases, it is safe to deprescribe anti-hypertensives to prevent the occurrence of hypotension, which is a higher risk at the end-of-life and can result in unintended adverse events, including dizziness and falls. Although, as an exception, discontinuing beta blockers is not appropriate as the aim to directed at heart rate rather than blood pressure management. In the hospice population, primary prevention of disease processes is not the goal, and for anti-hypertensives, the time to therapeutic benefit is longer than life expectancy (Thompson, 2019). In a parallel-group, unblinded clinical trial, anti-hypertensive medications were deprescribed in an older adult population having advanced disease. Concluding after the 16-week follow-up visit, there were no increased adverse events, although systolic blood pressure was elevated by a mean increase of $7.36 \mathrm{~mm} \mathrm{Hg}$ and diastolic blood pressure elevated by a mean increase of $2.63 \mathrm{~mm} \mathrm{Hg}$ (Moonen et al., 2015). Conclusions from Moonen's study, along with numerous others, indicated that appropriate discontinuing of anti-hypertensives can alleviate polypharmacy, pill burden, and adverse drug events in the patient with a limited prognosis.

Aspirin as a primary prevention strategy to reduce cardiovascular disease has been controversial in the last few years for older adults. Historically, aspirin has been prescribed daily for 40-70 years old to reduce the 10-year risk of myocardial infarction or ischemic stroke. Nevertheless, with advanced age over 70 years old in conjunction with multiple comorbidities, aspirin's continued use has been associated with significant bleeding risk (Selak et al., 2018). 
Concerning hospice patients specifically, continued use of primary prevention aspirin is considered insignificant as the long-term efficacy exceeds the life expectancy. In 2019, the American College of Cardiology (ACC) updated its guidelines and revoked the previous recommendation to use aspirin as primary prevention. Modifying the guidelines for routine aspirin use in the elderly population has changed because of multiple RCTs, meta-analyses, and high-quality cohort studies (Jacobsen et al., 2020). Such evidence has determined continuing aspirin therapy is associated with major hemorrhagic events in the elderly and does not reduce cardiovascular mortality or morbidity (Arnett et al., 2019).

\section{Methods}

Designed as a phased retrospective quality improvement project, there is potential for implementing a streamlined and consistent method to improve deprescribing within Sharp Hospice. This project can expand into an interventional evidence-based practice project by implementing a deprescribing protocol and educational plan. This project can be sustained as standard practice throughout Sharp Hospice with increased awareness regarding how many patients are accurately being deprescribed. This project's next crucial step will be providing education to the patients and families by assessing their baseline and willingness to learn more about how deprescribing and polypharmacy and clinician participation in routine deprescribing practices. Polypharmacy and deprescribing are new terms to many patients and their families, requiring extensive education to improve patient's agreement with removing medications during hospice enrollment or soon after that. Conversations with patients and their families, not once but repeatedly during the hospice services, are vital to reducing polypharmacy burden without misinterpreting medications' removal to hasten death. Once the education is delivered, deprescribing may be acknowledged as more helpful than harmful by patients and their families, 
DEPRESCRIBING IN THE HOSPICE POPULATION, WHO IS ELIGIBLE AND WHO IS AGREEABLE: A QUALITY IMPROVEMENT PROJECT

improving deprescribing rates. Patients and their families will conduct a pre-and post-

assessment, if cognitively intact and agreeable, determining if the education was impactful.

After determining sufficient evidence and completing an appraisal, it was concluded that medications used for risk reduction in long-term medical management are appropriate to deprescribe in the end-of-life period (Copper 2015). The original PICOT question was: "In newly enrolled hospice patients (P), will eligibility screening for medication deprescribing (I) result in discontinuation of target medications $(\mathrm{O})$ compared to usual care $(\mathrm{C})$ over four weeks (T)?’ Unfortunately, due to the COVID-19 pandemic, this PICOT question could not be entirely answered as the project design required modifications to adhere to isolation restrictions. It was decided to shift the project's focus to an early phase, noninterventional quality improvement project at Sharp Hospice to screen patients to determine who was eligible for target medication deprescribing and_capture data regarding current deprescribing practices. This necessary first step will provide accurate data to clinicians and administrators for deprescribing program planning in the subsequent phases of the project. After extensive literature review, the identified medications referred to as target medications in this project, can be deprescribed without increasing mortality (Deprescribing Network, 2020). Identified target medications included: antihypertensives, excluding medications for heart rate control, such as beta blockers, antihyperlipidemics, and diabetic medications, excluding insulins. The most common of these medications specific to Sharp Hospice included: Atorvastatin, Metformin, Amlodipine, Metoprolol, and Hydralazine. Removal of target medications in the four weeks succeeding hospice admission is defined as appropriate deprescribing.

In this project, patients were previously seeking complete medical treatment and, during a hospital admission, changed goals of care to comfort-focused hospice services. Although this 
specific inclusion criteria reduced the sample size, it was necessary to view the medication lists before and after initiating hospice. The inclusion criteria were patients who initiated hospice services directly after an inpatient admission at a Sharp hospital from August 01, 2020, to August 31, 2020, or discharged patients of the palliative care physician from the affiliated medical group. Exclusion criteria were patients who were previously enrolled in the Sharp palliative care "Transitions" program as these patients had a previous medication review before their hospice enrollment. This specific group of participants would have skewed the data resulting in a misinterpretation of results as many medications would have been previously deprescribed while in the Transitions program. Sharp hospitals are located primarily in southern San Diego County, yet Northern County has providers who also refer patients to Sharp Hospice. This specific data was retrieved but excluded from analysis due to the inability to view outside medical records. If there were the ability to access the medications administration records (MARs), this would have increased the project's sample size.

\section{Intervention}

Due to COVID-19 pandemic restrictions, the project's design required modification to a quality improvement rather than an interventional, evidence-based practice project. Originally the intervention was designed to target clinicians, including physicians and nurse practitioners, with evidence-based education for deprescribing but this approach was not permitted due to pandemic isolation restrictions. Instead, the project focused on the early phase of quality improvement - data collection and analysis to identify potential target goals for deprescribing. Identifying the eligibility of enrolled hospice patients provided valuable information regarding the scope of the problem and measured the gap in practice to set target goals for the program's next phase. 


\section{Measurable Outcomes}

The outcome measures are broken down into three major sections. The first section looks at the percent of target medications on the MAR while admitted to a Sharp facility. This initial measurable outcome set a baseline to determine the percentage of patients taking any of the target medications appropriate to be deprescribed immediately before hospice enrollment. Noting the percent of target medications remaining on the MAR at hospice admission is the second measurable outcome. This section of the data notes patients who are eligible for deprescribing target medications and capture how many are being deprescribed appropriately upon admission. Third, the percent of patients who still had the target medications on their MAR was noted four weeks after hospice admission. This data shows baseline deprescribing without any intervention to providers. In summary, the percentage of target medications at several time points was collected: before the hospice admission, after the hospice admission, and in the four consecutive weeks after hospice services have begun. This data is critical as it summarizes actual deprescribing rates and captures at what time point deprescribing was the highest in the sample.

\section{Data Collection}

Facilitators of this project included internal motivation and stakeholder support from Sharp Hospice, the ability to access the data, and a national deprescribing network providing specific guidelines. Barriers included data collection methods and organizational focus on the evolving pandemic. My clinical mentor retrieved data for the project to solve the limitation of students not being allowed on-site at Sharp Hospice. The second barrier included the organizational focus on patients and clinical support rather than initiating new projects. This resulted in a project design modification to prepare for implementation phases when Sharp Hospice can work on quality improvement projects. 
All participants admitted between August 01-August 31, 2020, were included if they had any of the target medications prescribed and had been admitted into Sharp hospice from a Sharp facility. An individual chart review of each patient's hospital discharge MAR was compared to the hospice admission medication list. A second medication review was completed four weeks after admission to determine if and when the target medications were discontinued. Basic demographic data, including age, gender, race, religion, and primary language was collected to gather information on what barriers may be associated with deprescribing and relevant demographical information. If the age exceeded 90 years old, the age was recorded as " $>89$ " to protect patient identity. Information regarding the location at the time of hospice referral, the hospice admission diagnosis, if discharged from the hospital with target medications, and specific target medications were recorded. Coding the subjects allowed for anonymous data collection, and no use personal identifiers were documented.

\section{Protection of Human Subjects}

Several organizations, including Sharp Hospice, Sharp Healthcare, and the University of San Diego, approved the project. The University of San Diego approved the project through the Institutional Review Board (IRB). After presenting the project methodology to the members of the Sharp Grossmont Hospital IRB team, Sharp Hospice's clinical director, vice president, and participating clinicians, it was approved. Privacy and confidentiality were protected by using a de-identified data set to analyze project outcomes. This data set will be kept for three years on a password-protected thumb drive, and results will be shared only in an aggregated manner. After three years, data will be destroyed. 
DEPRESCRIBING IN THE HOSPICE POPULATION, WHO IS ELIGIBLE AND WHO IS

AGREEABLE: A QUALITY IMPROVEMENT PROJECT

\section{Framework}

\section{DMAIC Framework}

As an organization, Sharp healthcare utilizes LEAN Six Sigma for a systematic approach for continuous and successful improvement. DMAIC is the LEAN Six Sigma problem-solving approach and stands for "Define, Measure, Analyze, Improve, and Control”. Applying this framework allowed for a systematic approach for a quality improvement project. The first step of DMAIC is to understand the scope of the problem; this is the essential step as it lays a foundation for a well-defined project that is easier to manage and meet projected outcomes. During participation in the weekly Sharp Hospice interdisciplinary team (IDT) meetings, improvement opportunities were defined as a lack of standardization regarding deprescribing. Measuring the data was completed through individual chart reviews and analyzing the data to determine percentages of deprescribing at specific time intervals. There was a significant amount of highquality evidence on deprescribing of specific medications in the hospice population. Due to project modifications, a root cause analysis could not be completed as it became an early phase noninterventional QI activity. Therefore, the improvement and control phases of the process were not completed. Moving forward into the subsequent phases of this project, completing a root cause analysis will determine what barriers hinder deprescribing. This will allow for a preand post-implementation data analysis to compare the volume of deprescribing. Lastly, the improvements should be sustainable and disseminated through Sharp Hospice providers and clinicians (Sharp: DMAIC, 2020).

\section{Change Acceleration Process Model}

The Change Acceleration Process Model is a set of tools to facilitate the acceleration of change through implementation within the organization. Sharp Healthcare uses CAP to assess 
the climate before any change has begun and increase the possibilities of successful change that is sustainable and quick. Creating this project with the COVID-19 pandemic restrictions in mind, it became apparent the ability to change deprescribing processes in Sharp Hospice would remain incomplete. However, CAP was used to design the project methodology and work toward completing deprescribing protocols to implement effectively and efficiently. Changes to a medication regimen can be daunting for patients and their families, especially during a time of such rapid changes. Deprescribing, although found to have multiple benefits, should be done with patient and family-centered decision-making in healthcare delivery. This model is imperative to use for the successful data dissemination to Sharp Hospice providers, which directly translates to how well the improvement of deprescribing will be (Change Acceleration Processes (CAP), n.d.).

\section{Meleis' Transitions Theory}

Meleis' Transition theory (2000) serves as a template for the multitude of concepts regarding change as patient move through different care settings. In this model, Meleis and colleagues outline several vital areas one must first understand to create a change that is not only feasible but sustainable. Awareness, the first step in the transition model, is a defining characteristic of any change, which is similarly true regarding shifting goals of care from complete medical treatment to hospice services. With understanding and awareness of the circumstances and inevitable bodily failure, hospice changes can be welcomed with ease compared to impediment by utilizing Meleis' Transition Theory. Deprescribing awareness is significant for patients' and families' positive support of the changes made to the medication list, and this is done so by engaging them in conversation throughout the hospice enrollment. Providing a simplified synthesis of the research supporting deprescribing and how beneficial it is 
for their loved one is more likely to lead to medication changes and minimize hesitation regarding risk of deprescribing. Timespan is critical to recognize. Successful change does not occur rapidly; hence, collecting data to capture how many patients are deprescribed in the first month of hospice admission will provide information determining if change is readily accepted in the first four weeks. Meleis' Transition Model serves as a guide to ease hesitation around deprescribing and create an achievable hospice transition for the patient and their family (Meleis et al., 2000).

\section{Holmes' Model of Deprescribing}

After reviewing several existing frameworks and guidelines, Holmes determined a need for all providers to consider deprescribing at every patient interaction (2015). The Holmes' Model of Deprescribing identified five recommendations for deprescribing in patients with limited life expectancy. The five recommendations include shared decision making, not prescribing medication as an option, deprescribing as a part of prescribing, embracing uncertainty, and the impact of difficult discussions. This model helps guide an understanding of how deprescribing is different with end-of-life patients and the unique challenges and associated barriers, which may be successfully overcome. (Todd \& Holmes, 2015). Using these recommendations in conjunction with the frameworks for successful change can lead to an effective implementation of deprescribing at Sharp Hospice. These recommendations add value to this phase of the project and benefit the application of intervention in the next phase.

\section{Results}

Data analysis revealed the percentage of deprescribing of target medications and allowed review of any missed opportunities for deprescribing. One hundred sixty-four patients were admitted to Sharp Hospice from August 01, 2020, to August 31, 2020. Of the total 164 patients, 
60 were automatically excluded from the project as their referral source was an outside agency other than Sharp Healthcare, resulting in the inability to access the inpatient MAR. A second exclusion included whether death occurred during the initial four weeks after hospice enrollment, which equated to $40 \%$ of the patients. The final number of eligible patients was 34 .

The first outcome was the percentage of target medications on the MAR while the patient had a hospital stay in a Sharp facility, as shown in Figure1. There were three target medication classes, of which equaling $80.5 \%$ were antihypertensives, $61.1 \%$ were statins, and $22.2 \%$ were oral hypoglycemic medications.

\section{Figure 1}

\section{Percentage of Target Medications Before Hospice Admission Eligible to Deprescribe}

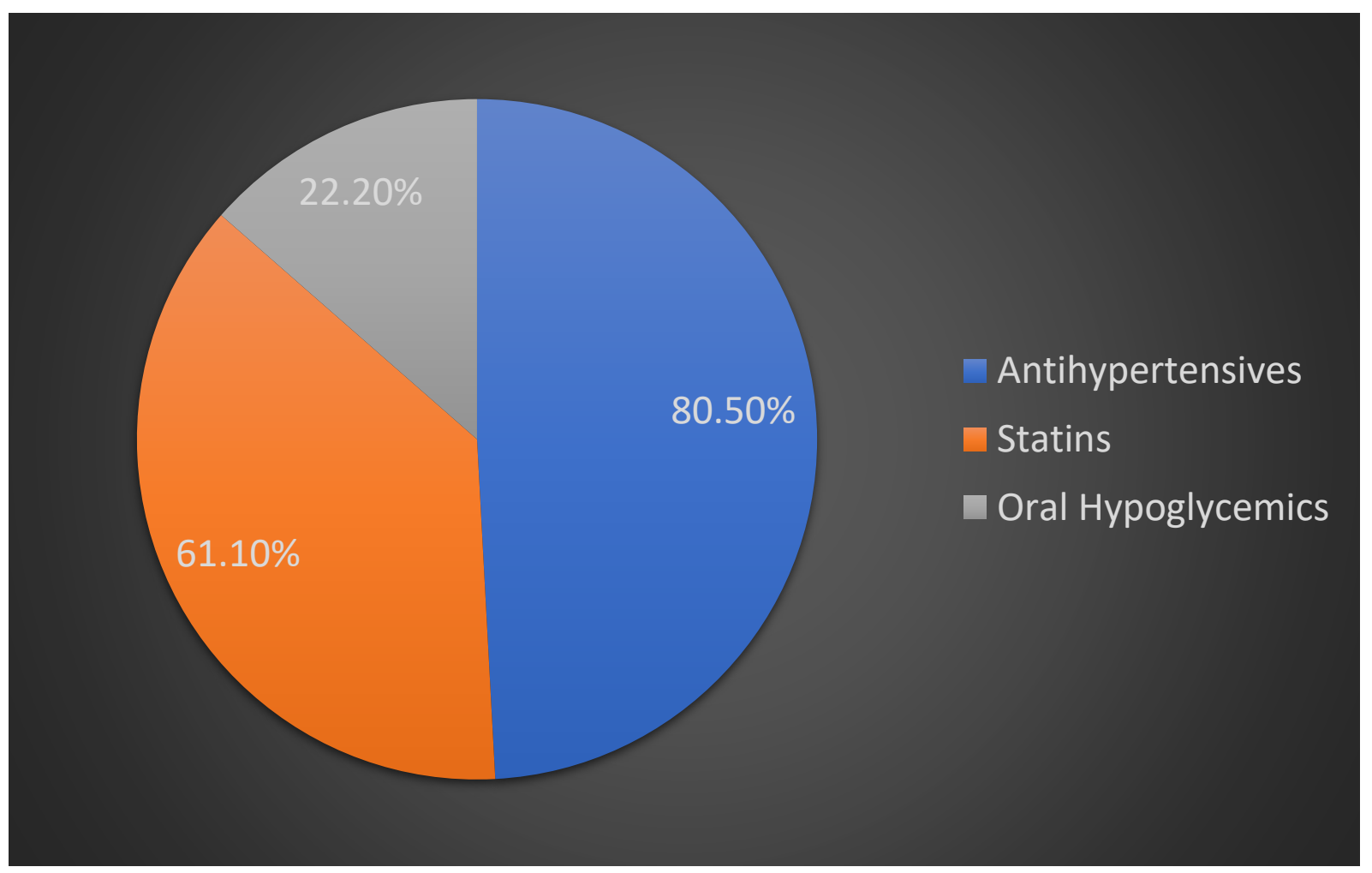

It was noted $61.76 \%$ of patients were taking at least two of the target medications, and

$14.70 \%$ of patients were taking three or more of the target medications eligible for deprescribing. Deprescribing was evaluated in three ways: fully, partially, or not at all deprescribed. Fully 
DEPRESCRIBING IN THE HOSPICE POPULATION, WHO IS ELIGIBLE AND WHO IS AGREEABLE: A QUALITY IMPROVEMENT PROJECT

deprescribed indicated discontinuation of all eligible medications from the MAR. Partially

deprescribed indicated only some of the eligible medications were deprescribed and not deprescribed indicated no removal of the eligible medications.

The second measurable outcome was to determine the percent of target medications remaining on the MAR at the hospice admission. $69.23 \%$ of patients were appropriately deprescribed at the time of hospice admission, $7.69 \%$ of patients were partially deprescribed, and $23.08 \%$ of patients were not deprescribed as shown in Figure 2. The percentage of change from before hospice admission to directly after admission was a $64 \%$ difference in removing eligible target medications from the MAR.

\section{Figure 2}

Deprescribing at Different Intervals

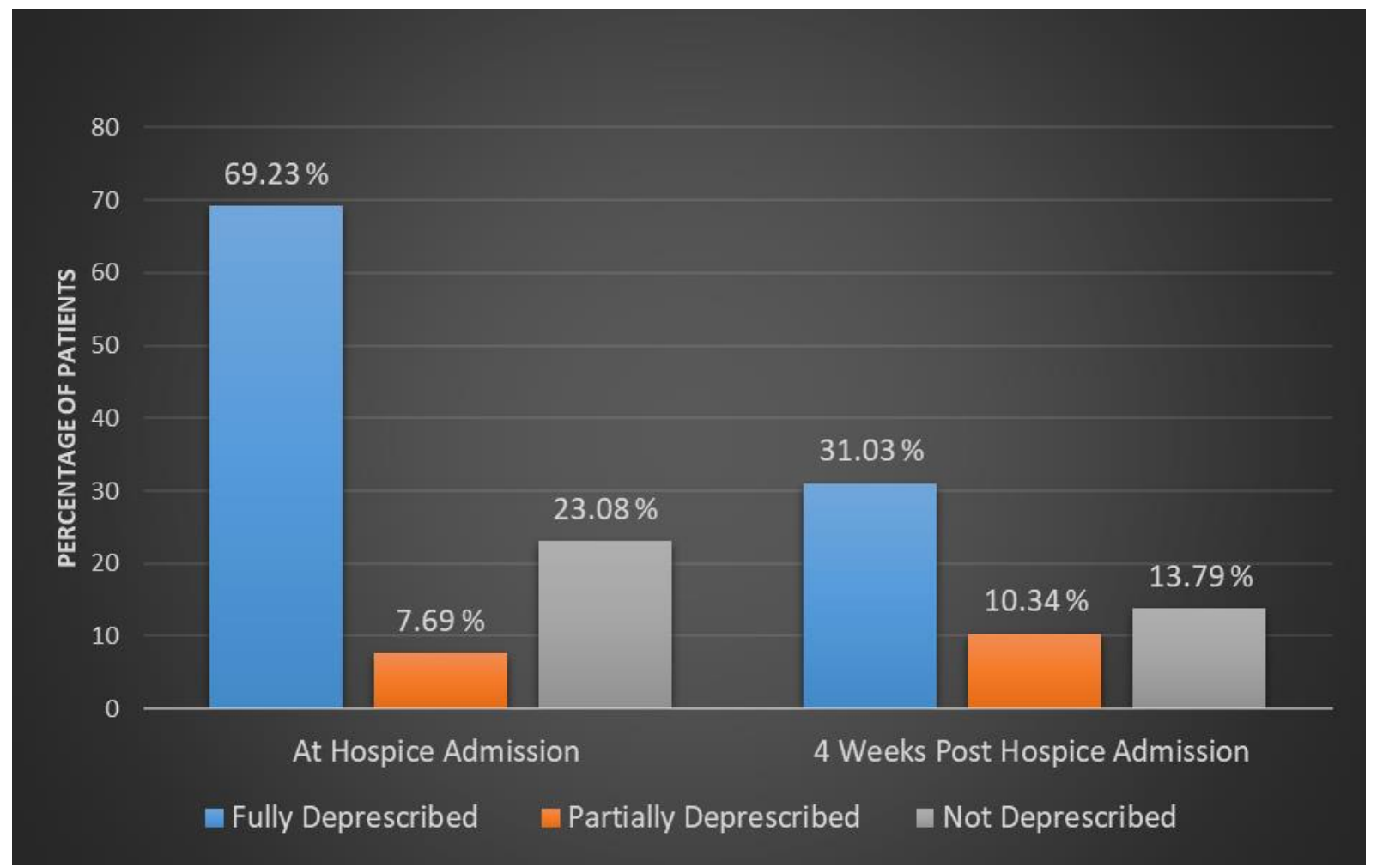


The third outcome measured was the percent of target medications on the MAR at four weeks post hospice enrollment. Of these, $31.03 \%$ had all target medication deprescribed, $10.34 \%$ had some target medication remaining on the MAR, and $13.79 \%$ had all the target medication remaining on the MAR from pre hospice enrollment. One explanation for the decrease at the four-week time is patients who died before that measurement point.

Analysis of the demographics revealed the mean age range was 77 years old; $61.7 \%$ were female, and $38 \%$ were male. The most prevalent race was Caucasian totaling $41.67 \%$, and the second was Hispanic at $28.57 \%$. Interestingly, there was only one Arab-American patient, which may reflect the impact of culture, race, religion, and primary language spoken on hospice deprescribing. There were three primary hospice diagnoses, including congestive heart failure (10.34\%), chronic obstructive pulmonary distress (10.34\%), and cancers collectively (20.58\%), as seen in Figure 3.

\section{Figure 3}

\section{Hospice Admission Diagnoses}

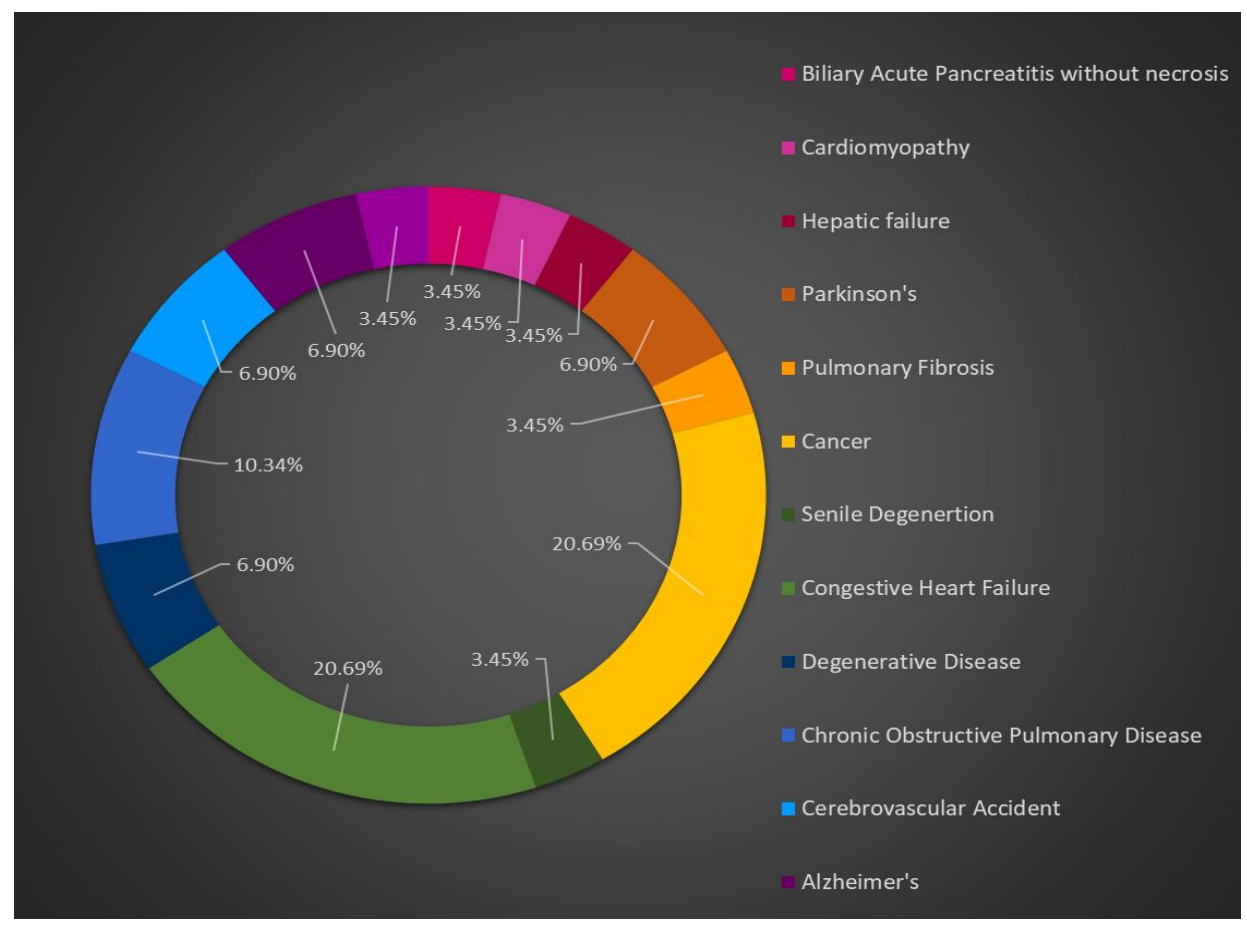




\section{Cost Analysis}

Due to the project design and remote interactions, there were no costs associated with the early phase of this project. Data was collected from existing admission and census reports. Although the target medications were not a direct organizational cost of Sharp Hospice, reduction of polypharmacy and appropriate deprescribing can have a substantial financial impact on healthcare costs. Of the 34 patients included in this project, $61.1 \%$ of patients were taking a statin. As an exemplar, the median cost for a 30-day supply of Atorvastatin is $\$ 18.00$ (Atorvastatin Prices, Coupons \& Patient Assistance Programs, 2021). Twenty-three patients were taking the medication multiplied by the monthly cost equaled to $\$ 414.00$ per month $(23 \times \$ 18.00$ $=\$ 414.00)$. A deprescribing protocol in place for this one medication alone could result in significant savings per month. Reducing out-of-pocket monthly medication costs is a significant facilitator for patients agreeing to deprescribing (Kutner, et al., 2015; Bukowy \& Teso, n.d.)

\section{Strength and Limitations}

This project's strength was an accurate depiction of how deprescribing is done in Sharp Hospice, determining baseline percentages of deprescribing completed by the providers. This baseline data is valuable information for project continuation and the interventional implementation component. Due to the clinical site being inaccessible because of pandemic restrictions and necessary organizational priorities, the project required redesigning from interventional to an early-phase quality improvement project. The major limitation of this project was the inability to complete an intervention to increase deprescribing as the pandemic restricted physical contact with patients, families, and Sharp Hospice providers. Another critical limitation to take into consideration was the small sample derived from only one hospice organization in a 
limited time period which may not be an accurate representation of other hospices or a longer measurement period.

\section{Implications}

The choice to deprescribe is not a simple decision; it is instead a complicated and openended obligation requiring careful examination of the patient as a whole. The finding of this project's early phase demonstrated appropriate deprescribing was completed yet found some medications eligible for discontinuation were not removed for undetermined reasons. The original PICOT question was unable to be unanswered, but this phase of the project allowed for a preliminary analysis of current deprescribing rates in Sharp Hospice. This information is valuable for understanding a baseline and plan for actions that will improve deprescribing in the subsequent implementation phase. For example, in the first four weeks of hospice enrollment, $40 \%$ of the patients eligible for the project died although this is a non-modifiable excluding factor. When designing the next phase of implementation this group of patients could benefit from immediate deprescribing upon hospice enrollment compared to a delay in deprescribing, as seen in the four-week post-enrollment analysis. Without this pre-phase implementation project, it would have been challenging to create a meaningful and successful project to improve deprescribing. This phased implementation may provide insight to other hospice agencies reviewing deprescribing in their practice. The early phase allowed determination of a baseline deprescribing rate and clarified what medications are most common to the specific patient population of Sharp Hospice. This information would be beneficial to know before implementing a standardized deprescribing protocol in Sharp Hospice or elsewhere. The specificities may differ in other regions, resulting in a need for modification and individualization of other agencies' project planning. 
Although hospice care primary objective is eliminating distress during the end-of-life period, polypharmacy may still occur, resulting in unwanted consequences for patients. Adverse drug events can be reduced or eliminated by accurately deprescribing when medically appropriate and necessary. A significant percentage of patients were taking at least two of the target medications. As mentioned earlier, there is a risk of adverse drug events occurring with medication combinations which can more readily induce the polypharmacy cascade. This analysis revealed that more than half of the organization's new patients were eligible for deprescribing. Sucessful future implementation could be realized after designing a systematic organizational program for deprescribing using DMAIC. An essential step for the interventional phase would educate patients and their families to provide a comprehendible rationale. Meleis' Transition Theory is also beneficial for application when providing education as it touches on the sensitive nature involved with changes and how barriers may be overcome (Meleis et al., 2000).

Other implications to consider are the various factors that might influence the patient's and family's approval for a plan to deprescribe. Some medications were deprescribed during the hospice admission yet not fully. This could have resulted from multiple factors such as race, religion, and primary language spoken. Demographic factors have historically been associated with differences in patients' preferences in medical care and may continue in similar attitudes for hospice care. Although demographic data was collected, it was beyond the scope of an evidenced-based practice project to determine an impact of demographics on deprescribing. Applying the Holmes Model of Deprescribing, a screen for patient's willingness to accept education regarding deprescribing should be included in the next phase of implementation in the pre-implementation data collection process (Todd \& Holmes, 2015). Unfortunately, it was not in the project's scope to capture this data, as it is difficult and complex with many variables 
influencing the patient's decisions. In the next phase of interventional implementation, it would be valuable to understand how demographics create barriers or facilitate deprescribing.

There are exceptions to deprescribing in the classes of target medications in this project, which is another important consideration. Of the 34 patients included, $80.5 \%$ of patients were receiving antihypertensive medications. Although noting different medication types in this class, all antihypertensives were grouped together for data collection feasibility due to the inability to conduct a thorough review of each patient's chart, because of site access restrictions. For example, most antihypertensive medications observed in this project included: angiotensin converting enzyme (ACE) inhibitors, beta blockers, and calcium channel blockers. Although all medications in this class have a blood pressure lowering effect, beta blockers are used consistently for heart rate control (Mann, 2020). Beta blockers, such as Metoprolol and Carvedilol, should remain prescribed to prevent uncontrolled and chaotic heart rate fluctuations in patients with atrial fibrillation. A substantial percentage of patients, $38.2 \%$, were taking one of these medications. Although classified initially as an eligible medication for deprescribing, when reviewed in depth removing the medication was determined to be not appropriate. This is a caveat for deprescribing antihypertensives, and the clinician must consider individual consideration of each patient before removing medications. These nuances and specific situations were not accurately captured in this project and would require a separate, more detailed patient record review and data collection process once organizational priorities have shifted from the ongoing pandemic.

Deprescribing is a safe way to reduce polypharmacy occurrences and continue to uphold the ethical principle of non-maleficence. DMAIC, the change acceleration process, and Holmes Model of Deprescribing require a thorough assessment of the current situation to implement a 
DEPRESCRIBING IN THE HOSPICE POPULATION, WHO IS ELIGIBLE AND WHO IS AGREEABLE: A QUALITY IMPROVEMENT PROJECT

successful project. Upon completion, several implications emerged, and these findings may be generalizable to other organizations as they are specific to the hospice population in general, not just Sharp Hospice. Although completing many of the project's activities was not possible in this phase, it created an unwavering foundation to build the subsequent implementation phase. This early phase may serve as a reference for other hospice agencies to help guide a sucessful deprescribing project plan.

\section{Conclusion}

Successful deprescribing starts with understanding evidence-based recommendations and organizational baseline data. Although there are deprescribing protocols and guidelines, a standardized, fully implemented guideline for each target medication class would improve deprescribing rates. The findings from this project demonstrate the lack of deprescribing in the hospice population. Further understanding and increased education to the geriatric population (hospice and non-hospice) would help improve the knowledge regarding deprescribing safety and perhaps increase the percentage of patients willing to have medications removed. The topic of deprescribing is frequently disregarded as a part of a medical visit, yet it is just as important as prescribing itself. This project illustrated who in hospice was eligible for deprescribing, who was adequately deprescribed, and existing gaps for improvement.

In conclusion, not every medication type could be deprescribed safely; it requires a skilled and experienced clinician to review each medication and determine continuation, dose reduction, or deprescription. Disseminating this baseline data to Sharp Hospice's clinicians and leaders for discussion and action planning will be included in the project's implementation phase. The next feasible step would be to assess and reduce the barriers hindering deprescribing and to create a meaningful program to reduce the burden of unnecessary medications for hospice 
patients. Although not originally planned, this phased project established a foundation for higher impact intervention project in the future. 
DEPRESCRIBING IN THE HOSPICE POPULATION, WHO IS ELIGIBLE AND WHO IS

AGREEABLE: A QUALITY IMPROVEMENT PROJECT

\section{References}

Arnett, D. K., Blumenthal R. S., Albert M. A., Buroker A. B., Goldberger Z. D., Hahn E. J., Himmelfarb C. D., Khera A., Lloyd-Jones D., McEvoy J. W., Michos E. D., Miedema M. D., Muñoz D., Smith, S. C., Virani, S. S., Williams, K. A., Yeboah, J., \& Ziaeian, B. (2019). 2019 ACC/AHA Guideline on the Primary Prevention of Cardiovascular Disease: A Report of the American College of Cardiology/American Heart Association Task Force on Clinical Practice Guidelines. Circulation, 140(11), e596-e646. https://doi.org/10.1161/CIR.0000000000000678

American Diabetes Association (2021). 12. Older Adults: Standards of Medical Care in Diabetes-2021. Diabetes Care, 44(Supplement 1), S168-S179. https://doi.org/10.2337/dc21-S012

Bukowy, E. A., \& Teso, K. (n.d.). Deprescribing at the End of Life: When Less is More. 47. https://ocpe.mcw.edu/sites/default/files/Deprescribing\%20at\%20End\%20Of\%20Life\%20 4-30-19.pdf

Chau, S. H., Jansen, A. P. D., van de Ven, P. M., Hoogland, P., Elders, P. J. M., \& Hugtenburg, J. G. (2016). Clinical medication reviews in elderly patients with polypharmacy: A crosssectional study on drug-related problems in the Netherlands. International Journal of Clinical Pharmacy, 38(1), 46-53. https://doi.org/10.1007/s11096-015-0199-8

Change Acceleration Processes (CAP). (n.d.). What is Six Sigma. Net. https://www.whatissixsigma.net/change-acceleration-processes/

Cooper, J. A., Cadogan, C. A., \& Patterson, S. M. (2015). Interventions to improve the appropriate use of polypharmacy in older people: A Cochrane systematic review. $B M J$ Open., 5. https://doi.org/10.1136/bmjopen-2015-009235 
Frechen, S., Zoeller, A., Ruberg, K., Voltz, R., \& Gaertner, J. (2012). Drug Interactions in Dying Patients. Drug Safety, 35(9), 745-758. https://doi.org/10.1007/BF03261971

Jacobsen A. P., Raber I., McCarthy C. P., Blumenthal R. S., Bhatt D. L., Cusack, R. W., Serruys, P.W.J.C., Wijns, W., \& McEvoy, J. W. (2020). Lifelong Aspirin for All in the Secondary Prevention of Chronic Coronary Syndrome. Circulation, 142(16), 1579-1590. https://doi.org/10.1161/CIRCULATIONAHA.120.045695

Jeffreys, E., R., A. D., (2015). Diabetes Management at the End-of-Life. Palliative Care Network of Wisconsin. https://www.mypcnow.org/fast-fact/diabetes-management-at-the$\underline{\text { end-of-life/ }}$

Kutner, J. S., Blatchford, P. J., Taylor, D. H., Ritchie, C. S., Bull, J. H., Fairclough, D. L., Hanson, L. C., LeBlanc, T. W., Samsa, G. P., Wolf, S., Aziz, N. M., Currow, D. C., Ferrell, B., Wagner-Johnston, N., Zafar, S. Y., Cleary, J. F., Dev, S., Goode, P. S., Kamal, A. H., ... Abernethy, A. P. (2015). Safety and benefit of discontinuing statin therapy in the setting of advanced, life-limiting illness: A randomized clinical trial. JAMA Internal Medicine, 175(5), 691-700. https://doi.org/10.1001/jamainternmed.2015.0289

Mann J.F.E. (2020). Choice of drug therapy in primary (essential) hypertension. Up To Date. https://www.uptodate.com/contents/search.

Masnoon, N., Shakib, S., Kalisch-Ellett, L., \& Caughey, G. E. (2017). What is polypharmacy? A systematic review of definitions. BMC Geriatrics., 17. https://doi.org/10.1186/s12877$\underline{017-0621-2}$

McLean, S., Sheehy-Skeffington, B., O’Leary, N., \& O’Gorman, A. (2013). Pharmacological management of co-morbid conditions at the end of life: Is less more? Irish Journal of Medical Science., 182. https://doi.org/10.1007/s11845-012-0841-6 
Meleis, AI., Sawyer, LM., Im E, Messias, DKH., \& Schumacher, K. (2000). Experiencing transitions: An emerging middle-range theory. Advances in Nursing Science, 23, (1), 1228. https://doi.org/10.1097/00012272-200009000-00006

Pruskowski, J. (2018). Fast Facts and Concepts \#321 Deprescribing. Palliative Care Network of Wisconsin. https://depts.washington.edu/fammed/wp-content/uploads/2018/12/FF-321-

\section{Deprescribing.docx}

Selak, V., Kerr, A., Poppe, K., Wu, B., Harwood, M., Grey, C., Jackson, R., \& Wells, S. (2018). Annual Risk of Major Bleeding Among Persons Without Cardiovascular Disease Not Receiving Antiplatelet Therapy. JAMA, 319(24), 2507-2520. https://doi.org/10.1001/jama.2018.8194

Schenker, Y., Kavalieratos, D., Resick, J., Park, S. Y., Jeong, K., Pruskowski, J., Abernethy, A., \& Kutner, J. S. (2019). Associations Between Polypharmacy, Symptom Burden, and Quality of Life in Patients with Advanced, Life-Limiting Illness. JGIM: Journal of General Internal Medicine, 34(4), 559-566. https://doi.org/10.1007/s11606-019-04837-7

Todd, A., \& Holmes, H. M. (2015). Recommendations to support deprescribing medications late in life. International Journal of Clinical Pharmacy, 37(5), 678-681. https://doi.org/10.1007/s11096-015-0148-6

Thompson, J. (2019). Deprescribing in palliative care. Clinical Medicine, 19(4), 311-314. https://doi.org/10.7861/clinmedicine.19-4-31 
